\title{
The semi-Euclidean approach in statistical mechanics. II. The cluster expansion, a special example*
}

\author{
Paul Federbush \\ Department of Mathematics, University of Michigan, Ann Arbor, Michigan 48104 \\ (Received 29 April 1975)

\begin{abstract}
A form of the Glimm-Jaffe-Spencer cluster expansion, adapted to the statistical mechanics setting, is shown to converge for certain two-body potential interactions. The theory treated corresponds to negatively charged fermions and positively charged bosons interacting by a modified Coulomb interaction-the $1 / r$ potential, cutoff at high and low momenta, becoming $(1 / r)\left(e^{-a r}-e^{-\gamma r}\right)$.
\end{abstract}

\section{INTRODUCTION}

In Ref. 1 a formalism was presented to adapt the semi-Euclidean approach from constructive quantum field theory to statistical mechanics. In the present paper the Glimm-Jaffe-Spencer cluster expansion ${ }^{2}$ is shown to have a close analog in this setting. The theory treated corresponds to negatively charged fermions and positively charged bosons interacting by a modified Coulomb interaction-the $1 / r$ potential, cutoff at high and low momenta, becoming $(1 / r)\left(e^{-\alpha r}-e^{-\gamma r}\right)$. For suitable values of the parameters the cluster expansion patterned after Ref. 2 will be shown to converge. We hope to come back in a later paper to the $\gamma=\infty$ case, whose treatment should require only technical improvements over the present procedure. The methods of Ref. 3 apply to the interaction treated in the present paper, but not to the $\gamma=\infty$ situation. We have no definite ideas on how to treat the physically interesting case with $\alpha$ $=0, \gamma=\infty$.

A knowledge of Ref. 2 is required to read the present paper. Space is cut into unit cubes-the greater flexibility of allowing other size cubes is sacrificed to agree most completely with Ref. 2. Before presenting further details we point out the following similarities and differences to orient the reader.

(a) The potential $(1 / \gamma)\left(e^{-\alpha r}-e^{-\gamma r}\right)$ is interpolated by exactly the same procedure as in Ref. 2.

(b) Unlike Ref. 2 we here interpolate Hamiltonians rather than covariances.

(c) The interaction portion of the Hamiltonian is interpolated as indicated in (a). The kinetic energy portion is interpolated by erecting parameter dependent barriersan infinite barrier giving Dirichlet data.

(d) Estimates of integrals of functions on Euclidean space are replaced by operator estimates from Ref. 1, characteristic of the semi-Euclidean approach.

We anticipate extension of the present work to more general interactions, as well as the development of a cluster expansion for boson-fermion field theory models within the semi-Euclidean formalism.

\section{THE CLUSTER EXPANSION}

Space is filled with unit cubes $\left\{\Delta_{i}\right\}$. Each $\Delta_{i}$ is of the form $a_{i} \leqslant x_{i} \leqslant a_{i}+1, i=1,2,3$ for some set of integers $\left(a_{1}, a_{2}, a_{3}\right)$. A union of such cubes is said to be intimately connected if it remains connected after removal of all edges and vertices (i. e., contacts must take place across faces). The set of faces is denoted by $\left\{F_{i}\right\}$, the indexing of $\Delta_{i}$ and $F_{i}$ being unrelated. If $W$ is a union of cubes, we define

$$
\hat{W}=\{\mathbf{x} \in W \mid d(\mathbf{x}, \partial W) \geqslant \delta\}
$$

for a small parameter $\delta$.

With a given $W$ as above, we associate the Foch Hilbert space, $H_{w}$, constructed of functions vanishing outside $\hat{W} \cdot \operatorname{Tr}_{W}$ is the trace over this Hilbert space. Our interaction will be made up of Yukawa interations $e^{-n r} / r$, in the infinite volume. For a union of cubes $W$ we define $y_{W}^{n}(x, y), x$ and $y$ in $W$, by

$\left(-\Delta+n^{2}\right)_{x} y_{W}^{n}(x, y)=\left(-\Delta+n^{2}\right)_{x} \exp (-n|x-y|) /|x-y|$

and

$$
y_{W}^{n}(x, y)=0 \text { if } y \in \partial W .
$$

Note that

$$
y_{R^{3}}^{n}(x, y)=\exp (-n|x-y|) /|x-y| .
$$

With a union of cubes $W$, we associate $\Delta_{W}$, the Laplacian on functions defined in $\hat{W}$ satisfying Dirichlet data on $\partial \hat{W} . \Lambda$ is a fixed finite intimately connected union of cubes. $\bar{\psi}, \psi$ and $\bar{\phi}, \phi$ are fields describing fermions and bosons, respectively. $H=H^{\Lambda}$ is the Hamiltonian defined on $H_{\Lambda}$ :

$$
\begin{aligned}
& H^{\Lambda}=H_{0}^{\Lambda}+V^{\Lambda}, \\
& H_{0}^{\Lambda}=-\frac{1}{2 M} \bar{\psi} \Delta_{\Lambda} \psi-\frac{1}{2 m} \bar{\phi} \Delta_{\Lambda} \phi+\frac{\mu}{\beta} \bar{\psi} \psi+\frac{\mu}{\beta} \bar{\phi} \phi, \\
& V^{\Lambda}=\frac{1}{2}:(\bar{\psi} \psi-\bar{\phi} \phi) v^{\Lambda}(\bar{\psi} \psi-\bar{\phi} \phi):, \\
& v^{\Lambda}=q^{2}\left(y_{\Lambda}^{\alpha}-y_{\Lambda}^{\gamma}\right) .
\end{aligned}
$$

Integrals have been suppressed. If it is clear, the $\Lambda$ indicators may be omitted. For any union of cubes, $W$ say, a similar expression $H^{W}$ may be constructed. Using the notation of Ref. 1, we want to consider an expression of the form

$$
\operatorname{Tr}_{\Lambda}\left[T \exp \left(-\int_{0}^{\beta} H^{\Lambda}(t) d t\right) A\right]
$$

with $A$ a polynomial in the fields. For simplicity we will also assume all the fields in $A$ localized in a fixed cube $\Delta_{0}$. 
We now proceed to consider the interpolated Hamiltonians. A chanracteristic function for a neighborhood of the face $F_{i}$ is defined as follows:

$$
\chi_{i}=\left\{\mathbf{x} \mid d\left(\mathbf{x}, F_{i}\right)<\delta\right\}
$$

With each $F_{i}$ is associated a parameter $s_{i}, 0 \leqslant s_{i} \leqslant 1$. We define

$$
h(s)=(1 / s)-1
$$

so that $h(0)=\infty$ and $h(1)=0$. The barrier potentials are given by

$$
\begin{aligned}
& B_{s}=\sum B_{i s}, \\
& B_{i s}=(\bar{\psi} \psi+\bar{\phi} \phi) \chi_{i} h\left(s_{i}\right) .
\end{aligned}
$$

$v_{s}$ is $v$ interpolated by the same definition as in Ref. 2. This makes sense since $\exp (-n r) / r$ has a Fourier transform with the same form as a covariance. Thus we have

$$
V_{s}^{\Lambda}(t)=\frac{1}{2}:(\bar{\psi} \psi-\bar{\phi} \phi) v_{s}^{\Lambda}(\bar{\psi} \psi-\bar{\phi} \phi): .
$$

Time dependences are introduced as in Ref. 1. Finally we have the interpolating Hamiltonians:

$$
H_{s}(t)=H_{0}(t)+V_{s}(t)+B_{s}(t) .
$$

Note $H_{s}(t)=H(t)$ if, for all $i, s_{i}=1$.

We are now set to define the cluster expansion. For any union of cubes $X$ define

$$
X^{c}=\Lambda-X
$$

and $Z_{w}$ by

$$
Z_{W}=\operatorname{Tr}_{W}\left[\exp \left(-\beta H^{W}\right)\right] \text {. }
$$

Our substitute for $(3,15)$ of Ref. 2 is the following:

$$
\begin{aligned}
\operatorname{Tr}_{\Lambda} & {\left[T \exp \left(-\int_{0}^{\beta} H^{\Lambda}(t) d t\right) A\right] / Z_{\Lambda} } \\
= & \sum_{\substack{X \mathfrak{i}_{\circ}, C_{X} \\
\Delta}} \int \partial^{\Gamma} \operatorname{Tr}_{X}\left[T \exp \left(-\int_{0}^{\beta} H_{s}^{X}(t) d t\right) A\right] d s(\Gamma) \cdot Z_{X} c / Z .
\end{aligned}
$$

The notation $i_{0} c$, means that $X$ must be intimately connected. $\Gamma$, as in Ref. 2 , is a subset of the faces in the interior of $X$, such that the faces not in $\Gamma$ but in the interior of $X$ do not separate the interior of $X$. The result we claim is that for fixed $\beta, m, M$, and $\gamma-\alpha$, if $\mu$ and $\alpha$ are large enough and $g^{2}$ and $\delta$ are small enough then (1.18) converges uniformly in the volume $\Lambda$. From this result follows analogs of all the results in Ref. 2 .

\section{UNIFORM STABILITY OF THE POTENTIALS}

The two-body potential $v(x, y)$ is said to be stable if for any set of $N$ points $\left\{x_{i}\right\}$

$$
\sum_{i<j}^{N} v\left(x_{i}, x_{j}\right) \geqslant-C N
$$

for some constant $C$ independent of $N$ and the $\left\{x_{i}\right\}$. Applying this definition to our problem, we claim there is a constant $L$ such that

$\frac{1}{2} \iint_{W} d x d y:(\bar{\psi} \psi-\bar{\phi} \phi)(x) v_{s}^{W}(x, y)(\bar{\psi} \psi-\bar{\phi} \phi)(y):$

$$
\geqslant-L q^{2} \int_{w} d x(\bar{\psi} \psi+\bar{\phi} \phi)(x) .
$$

We say the $v_{s}^{W}$ are uniformly stable. $L$ is independent of $W, q$, and $\left\{s_{i}\right\}$. In fact, $L$ can be picked equal to $\frac{1}{2}(\gamma-\alpha)$. Without loss of generality we set $q=1$ in the remainder of this section.

For orientation we consider the free case, $W=R^{3}$ and $s_{i}=1$, all $i$. Then $v=\exp (-\alpha|x-y|) /|x-y|$ $-\exp (-\gamma|x-y|) /|x-y|$. There follows

$$
\begin{aligned}
\frac{1}{2} \iint & d x d y:(\bar{\psi} \psi-\bar{\phi} \phi) v(\bar{\psi} \psi-\bar{\phi} \phi): \\
= & \frac{1}{2} \iint d x d y(\bar{\psi} \psi-\bar{\phi} \phi) \int \frac{d k}{2 \pi^{2}} \exp (i k \cdot x) \exp (-i k \cdot y) \\
& \times\left(\frac{1}{k^{2}+\alpha^{2}}-\frac{1}{k^{2}+\gamma^{2}}\right)(\bar{\psi} \psi-\bar{\phi} \phi) \\
& -\frac{1}{2} \int d x(\bar{\psi} \psi+\bar{\phi} \phi)(x)(\gamma-\alpha) \\
\geqslant & -\frac{1}{2}(\gamma-\alpha) \int d x(\bar{\psi} \psi+\bar{\phi} \phi)(x) .
\end{aligned}
$$

We have used the fact that a positively weighted integral of operators times their conjugates is positive.

In the general case $v_{s}^{w}$ is constructed as a positively weighted sum, with total weight one, of $\left\{v_{\widetilde{s}}^{w}\right\}$, in which the values of the $s_{i}$ are restricted to one and zero. It is sufficient to prove the result for a single $v_{\widetilde{s}}^{W}$. Say the $s_{i}$ in $\tilde{s}$ equal to zero are those for which $i \in I$. Let $\left\{\phi_{j}\right\}$ be the set of eigenvectors of $\Delta$ in $W$ satisfying Dirichlet data on $\partial W$ and $F_{i}$ for $i \in I$ :

$$
\begin{aligned}
& \Delta \phi_{j}=-\lambda_{j} \phi_{j}, \\
& \phi_{j}=0 \text { on } \partial W \cup\left\{F_{i} \mid i \in I\right\} .
\end{aligned}
$$

Assume the $\phi_{f}$ are normalized:

$$
\int_{w} \phi_{j}^{2}=1
$$

Then

$$
v_{\tilde{s}}^{W}=4 \pi \sum_{j} \phi_{j} \phi_{j}\left(\frac{1}{\lambda_{j}+\alpha^{2}}-\frac{1}{\lambda_{j}+\gamma^{2}}\right)
$$

and

$$
\begin{gathered}
\frac{1}{2} \iint_{w} d x d y:(\bar{\psi} \psi-\bar{\phi} \phi) v_{\widetilde{s}}^{w}(\bar{\psi} \psi-\bar{\phi} \phi): \\
\geqslant-\frac{1}{2} \int_{w} d x(\bar{\psi} \psi+\bar{\phi} \phi) v_{\widetilde{s}}^{w}(x, x)
\end{gathered}
$$

as in (2.3). There follows

$$
\geqslant-\frac{1}{2} \int d x(\bar{\psi} \psi+\bar{\phi} \phi) \cdot \sup _{x} v_{\widetilde{s}}^{W}(x, x) .
$$

We are reduced to estimating $v_{\tilde{s}}^{W}(x, x)$ :

$$
v_{\tilde{s}}^{W}=\frac{4 \pi}{-\Delta+\alpha^{2}}-\frac{4 \pi}{-\Delta+\gamma^{2}}
$$

for the Laplacian satisfying the data in (2.4):

$$
=4 \pi \int_{0}^{\infty}\left[\exp \left(-\alpha^{2} t\right)-\exp \left(-\beta^{2} t\right)\right] \exp (\Delta t) d t
$$

So

$$
\begin{gathered}
v_{\tilde{s}}^{W}(x, x)=4 \pi \int_{0}^{\infty}\left[\exp \left(-\alpha^{2} t\right)-\exp \left(-\beta^{2} t\right)\right] \int d \mu_{x, x}^{t} d t, \\
\text { paths }(W, s),
\end{gathered}
$$


where $d \mu_{x, x}^{t}$ is the path space measure constructed for mass $1 / 2$ and the paths summed over lie in $W$ and avoid $\partial W$ and $\cup_{i \in I} F_{i}$. Clearly this is $\leqslant$ the value of the sum over all paths. So

$$
v_{s}^{2}(x, x) \leqslant \gamma-\alpha .
$$

\section{A GRAND CANONICAL ESTIMATE}

We define local number operators $N_{i}$ :

$$
N_{i}=\int_{\Delta_{i}}(\bar{\psi} \psi+\bar{\phi} \phi) d x \text {. }
$$

For a set of integers $\left\{\alpha_{i}\right\}$ we want to estimate

$$
G(\alpha)=\operatorname{Tr}_{w}\left[\exp \left(-\beta H_{0}^{W} / 2\right) \prod_{i} N_{i}^{\alpha} e^{N}\right]
$$

We claim if $|W|$ is the volume of $W$, and if $\mu$ is large enough, there is a $c_{1}$ such that

$$
G(\alpha) \leqslant\left(\prod_{i} \alpha_{i}^{\alpha_{i}}\right) \cdot \exp \left(c_{1}|W|\right) .
$$

Letting $D(z, V, T)$ be the grand canonical ensemble partition function, we see

$$
\begin{aligned}
D & =\sum_{0}^{\infty} z^{N} Q_{N}(V, T) \\
& =\left(z e^{2}\right)^{N} Q_{N}(V, T) \exp (-N) \exp (-N) \\
& =\left(z e^{2}\right)^{N} Q_{N}(V, T)\left(\prod_{i} \exp \left(-N_{i}\right)\right) \exp (-N) .
\end{aligned}
$$

(3.3) follows from

$$
N_{i}^{\alpha i} \exp \left(-N_{i}\right) \leqslant \alpha_{i}^{\alpha_{i}}
$$

and $D\left(z e^{2}, V, T\right) \leqslant \exp \left(c_{1} V\right)$ for $z$ small enough.

\section{CONVERGENCE}

Convergence is achieved basically the same way as in Sec. 10 of Ref. 2. In (10.1) of Ref. 2 one must estimate

$$
\int \partial^{\Gamma} \Pi_{i}^{n} \phi\left(x_{i}\right) \exp [-\lambda V(\Lambda)] d \phi_{s(\Gamma,} d s(\Gamma)
$$

we have been led to consider

$$
\int \partial^{\mathrm{r}} \operatorname{Tr}_{X}\left[T A \exp \left(-\int_{0}^{\beta} H_{s}^{X}(t) d t\right)\right] d s(\Gamma)
$$

To compare the two expressions note:

(a) The $\Lambda$ in (4.1) is an arbitrary volume, and the use of $X$ instead of $\Lambda$ would have been clearer.

(b) Our $A$ abbreviates $\Pi_{i}^{n} \phi\left(x_{i}\right)$ (rather an essentially equivalent expression); in fact, for the sake of convergence we can pick $A=1$ as this factor enters unessentially.

(c) The trace substitutes for the Euclidean space integration $\int d \phi_{s(\Gamma)}$.

The differentiations $\partial^{\Gamma}$ are performed in (4.2) using (2.2) of Ref. 1. Thereafter, a polynomial in the fields is downstairs in the trace. We now use (2.3) of [1] to move the fields according to the following steps.
Step 1: One at a time move each annihilation field $\phi$, $\psi$ to the right [using (3.2) of Ref. 1 to re-enter at left] until either it contracts with a creation field, or moves without contracting from $t=\beta$ to $t=\beta / 2$, in which case stop the field at $t=\beta / 2$.

Step 2: One at a time move each creation field $\bar{\phi}, \bar{\psi}$ to the left until either it contracts (with one of the annihilation fields at $t=\beta / 2$ ), or else having passed $t=0$ just once, stop the field at $t=\beta / 2$, to the left of all annihilation fields at $t=\beta / 2$.

At the end of this finite, noninductive, process all the fields downstairs in the trace are at $t=\beta / 2$, with creation fields all to the left of annihilation fields. With a field that has moved from $\left(x_{1}, t_{1}\right)$ to $\left(x_{2}, t_{2}\right)$ and then contracted (for simplicity without passing $t=0$ ) there is associated

$$
\int d \mu_{x_{1}, x_{2}}^{\left(t_{2}-t_{1}\right)}
$$

With a field $\bar{\phi}$ that has moved from $x_{1}, t_{1}$ left to reentry at $t=0$, and then brought to rest at $t=\beta / 2$ at $\left(x_{2}, \beta / 2\right)$ there is associated

$$
\int d w \int d \mu_{x_{1}, w}^{\left(\beta-t_{1}\right)} \int d \mu_{w, x_{2}}^{B / 2} \bar{\phi}\left(x_{2}, \beta / 2\right) .
$$

These are representative of all possibilities. As in Ref. 2 , we localize-but only the space variables. Thus for (4.3) there are two localization indices $\left(j_{1}, j_{2}\right)$. That is $x_{1} \in \Delta_{j_{1}}, x_{2} \in \Delta_{j_{2}}$. For (4.4) there are three localization indices $\left(j_{1}, j_{2}, j_{3}\right)$, with $x_{1} \in \Delta_{j_{1}}, w \in \Delta_{j_{2}}, x_{2} \in \Delta_{j_{3}}$.

There are now sums (over $j$ 's, partitions of $\Gamma$, and contractions) and integrals (over s's, $x^{\prime}$ 's, $t$ 's, and path space), the portion of the integrand we now consider is of the form

$$
\operatorname{Tr}_{w}\left(E_{1} \stackrel{s}{\Pi} \bar{\psi}\left(x_{i}\right) \stackrel{s}{\Pi} \psi\left(v_{i}\right) E_{2}\right) .
$$

Here $E_{1}$ and $E_{2}$ are time-ordered exponentials from $\beta / 2$ to $\beta$ and from 0 to $\beta / 2$ respectively. The $\bar{\psi}$ 's ( $\psi$ 's) stand for $\bar{\psi}$ 's and $\bar{\phi}$ 's ( $\psi$ 's and $\phi$ 's). In doing estimates we will take absolute values of the integrands, and use

$$
\begin{aligned}
& \left|\operatorname{Tr}_{W}\left(E_{1} \stackrel{s}{\Pi} \bar{\psi}\left(x_{i}\right) \stackrel{s}{\Pi} \psi\left(y_{i}\right) E_{2}\right)\right| \\
& \quad \leqslant \frac{1}{2} \operatorname{Tr}_{W}\left(E_{1} \stackrel{s}{\Pi} \bar{\psi} \psi\left(x_{i}\right) E_{1}^{*}\right)+\frac{1}{2} \operatorname{Tr}_{W}\left(E_{2}^{*} \stackrel{s}{\Pi} \bar{\psi} \psi\left(y_{i}\right) E_{2}\right) .
\end{aligned}
$$

Of these terms, that can be estimated alike, we consider the first term. All the $\vec{\psi}\left(x_{i}\right)$ in this term have associated to them, by (4.4), a path space integral

$$
\int d \mu_{w_{i}, x_{i}}^{B / 2}
$$

From the integrals to be performed we isolate the following portion of present interest:

$$
\operatorname{Tr}_{W}\left[E_{1} \prod_{i=1}^{s}\left(\int_{\Delta_{j_{3}}} d x_{i} \int d \mu_{w_{i}, x_{i}}^{\beta / 2} \bar{\psi} \psi\left(x_{i}\right)\right) E_{1}^{*}\right] \text {. }
$$

We have used the key fact, largely motivating our development to now, that $E_{1}$ does not depend on the integrals in (4.7). There are $c_{2}$ and $c_{3}$ such that

$$
\sup _{\substack{w_{i} \in \Delta_{j_{j_{i}}} \\ x_{i} \in \Delta_{j_{3_{i}}}}} \int d \mu_{w_{i}, x_{i}}^{\beta / 2} \leqslant c_{2} \exp \left[-c_{3} d\left(\Delta_{j_{2_{i}}}, \Delta_{j_{3_{i}}}\right)\right] .
$$


(Exponential falloffs are good enough for us; we sacrifice the actual Gaussian falloff.) Using (4.7) from Ref. 1 and $(3.3)$, we find that $(4.8)$ is less than

$$
c_{2}^{s} \exp \left(-c_{3} \sum_{i} d\left(\Delta_{j_{2_{i}}}, \Delta_{j_{3_{i}}}\right)\right) \exp \left(c_{1}|W|\right) \prod_{i} \alpha_{i}^{\alpha_{i}} \exp \left(c_{5}|\Gamma|\right)
$$

where $\Pi_{i} \alpha_{i}^{\alpha} i$ is the product over squares $\Delta_{i}$, and $\alpha_{i}$ is the number of $j_{3}$ 's in $\Delta_{i}$. By changing the free parameters as stated at the end of Sec. $1, c_{2}$ and $c_{5}$ can be made arbitrarily small and $c_{3}$ arbitrarily large. $c_{1}$ is not chosen to vary. The factor $e^{N}$ in (3.2) and $\exp \left(c_{5}|\Gamma|\right)$ arise as estimates of the exponent

$$
H(t) \geqslant \frac{1}{2} H_{0}-(N+8|\Gamma|) \frac{1}{2}(\gamma-\alpha) g^{2}
$$

using the uniform stability of the interaction. $8|\Gamma|$ is an estimate of the maximum number of "particles" added to the exponent by (2.3) of Ref. 1. We have kept $\frac{1}{2} H_{0}$ in (4.11) rather than $H_{0}$ to anticipate a development for more general interactions.

Prop.5.1, Prop.5.2, and Prop. 8.1 (for $v_{s}^{W}$ ) are the same here as in Ref. 2. The completion of estimates for convergence are parallel to those in Ref. 2 , Sec. 10. There is a mild novelty in the treatment of the barrier potentials.

\section{ACKNOWLEDGMENT}

I would like to thank Professor J. Rauch for the idea of interpolating Dirichlet data by barrier potentials and Professor B. Simon for forcing me to read about the cluster expansion.

*This work was supported in part by NSF Grant GP-17523. ${ }^{1}$ P. Federbush, J. Math. Phys. 16, xxxx (1975).

${ }^{2} \mathrm{~J}$. Glimm, A. Jaffe, and T. Spencer, "The Cluster Expansion," in Constructive Quantum Field Theory, The 1973

"Ettore Majorana" International School of Mathematical Physics, edited by G. Velo and A. Wightman (SpringerVerlag, Berlin, 1973).

${ }^{3} \mathrm{~J}$. Ginibre, "Some Applications of Functional Integration in Statistical Mechanics," in Statistical Mechanics and Field

Theory, Les Houches 1970 , edited by C. DeWitt and R. Stora (Gordon and Breach, New York, 1971). 